\title{
Behind the Masks of Bravery: A Phenomenological Study on the Challenges and Adjustments of Filipino Frontliners in Qatar
}

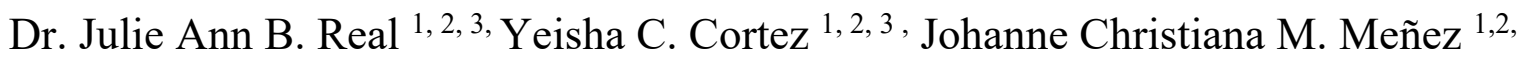 \\ 3, Hannah Rose D. Avendaño 1,2,3, Hannah Gwen B. Maquinaña 1,2,3, Silas Drew E. \\ Reyes ${ }^{1,2,3}$, Kyle P. Solivet ${ }^{1,2,3}$, Allen Bernard E. Nonato ${ }^{1,2,3}$ \\ 1 Philippine School Doha, Doha, Qatar \\ 2 Research Development, Accreditation and Publication Office, PSD, Doha, Qatar \\ 3 Research Capstone Project, PSD, Doha, Qatar
}

\begin{abstract}
The increasing cases of CoronaVirus (COVID-19) around the world have hugely affected the lives of families, workers, children, the government, and the community. This research aimed to understand the lived experiences of Filipino frontliners as Overseas Filipino Workers (OFW) in Qatar and how they deal with stress caused by their jobs as their coping mechanisms. This study utilized qualitative phenomenological research to deal with the common challenges dealt by frontliners. The data were gathered through a semi-structured interview with Filipino front-line workers who have been working in Qatar. The result shows that there were three key themes emerged in this research which are Work Constraints, Work Impact, and Coping Strategies. Frontliners experienced work constraints in their work field such as physical discomfort, emotional distress, and mental restraints. The work constraints mentioned have affected the front-line workers in their performance efficiency, individual being, and productivity enhancement. As a result, each frontliners developed their own coping strategies grouped as control management, adjustment measures, and prevention strategies. Frontliners bring risks to their own lives since they have to sacrifice a lot of their time for themselves, for their family, and for other people. Although front liners deal with a lot of stress and pressure in their work field, it is indeed rewarding to the Filipino front liners to be able to help the community. Being a frontliner is a tough however heroic job, thus maintaining a healthy body through regular exercise, resting, and eating healthy meals are crucial. Frontline employees also need the support and comfort they require from their loved ones by knowing that their family and friends are present for them. They will also be able to perform at their best if their responsibilities are assigned fairly, work hours are followed, and proper rests are provided.
\end{abstract}

Keywords: Frontliner; Phenomenology; Global Health; Work; Stress; Qatar

\section{Introduction}

In the event of a pandemic, the community needs people to defend them in dangerous situations. Frontliners, in general, are essential workers who work in hazardous environments. They operate in an exposed situation and provide regular and critical services. They are the first to respond in an emergency and those who, on a daily basis, put their lives on the line, especially during pandemics. According to Buhat (2020), because of the nature of frontliners' profession, which requires them to work in close quarters and interact with the public regularly, they are more likely to become infected. In relation to that, Sritharan et al. (2020) reported that 
frontline workers had the following mental health symptoms: fear, stress, anxiety, depression, insomnia, burnout, psychological and distress. These outcomes mainly came from COVID- 19, the effect of the pandemic. Risking their loved ones and themselves made the frontliners feel the symptoms which also affects their work performance.

Frontline workers are the ones who the community relies on the most. The responsibility they hold during a global pandemic is noble and worth recognition. Gamio (2020) stated that when responding to a probable coronavirus case, paramedics throughout the country are taking extra precautions. By doing so, frontliners carry the responsibility of taking secured measures to not spread the virus more. Despite being put under immense pressure, frontliners still carry a sense of duty to help and protect people.

This study describes the lived experiences of the challenges and adjustments of the Filipino frontliners in Qatar. The everyday lives of these frontline workers are shaped by their work schedules, presenting difficulties and necessitating the creation of coping mechanisms. This study aims to answer the central question: "What are the experiences of Filipino frontliners in the State of Qatar?", with the specific question: "How do Filipino frontliners deal with stress caused by their jobs and their coping mechanisms in Qatar?"

The research questions are formulated to narrow down the main inquiry to extract more specific responses. The questions revolved around the frontline workers' job environment and how it affects their physical, mental, and emotional well-being.

\section{Method}

\subsection{Research Design}

The qualitative phenomenological research method was used in this study. Crossman (2020) explained that qualitative research is a type of social science research that collects information and employs various data gathering methods such as interviews to focus on people's everyday lives and experiences. Moreover, research is a thorough examination of a topic, which frequently requires understanding others to get new insights into a phenomenon (Neubauer, Witklop, \& Varpio, 2019). Bloor and Wood (2006) also explained that the objective of the phenomenological technique is to describe, comprehend, and interpret the significance of human experiences.

A phenomenological approach was used in this study, because it fulfills the need to understand the adjustments and challenges experienced by the Filipino frontliners. The key issue in the research study focused on the various perspectives and adaptations of Filipino frontliners in the State of Qatar. The method of gathering the required data was collected and achieved through online interviews, and the gathered data was handled and analyzed to produce the themes found in the simulacrum. The questions utilized in the interview revolved around the common challenges faced by the frontliners due to stress and pressure, the effects of these challenges, and the adjustments made by the frontline workers to different work constraints.

The research study aimed to describe how stress and pressure affected the participants' personal and professional lives. This study assured a bias-free, open-ended method and stayed alert to any potential causes of failure. The objective of this research is to have profound and extensive knowledge concerning the experiences of Filipino frontliners. 


\subsection{Research Locale and Sample}

This research study took place at Philippine School Doha (PSD). The school is located in Doha, Qatar.

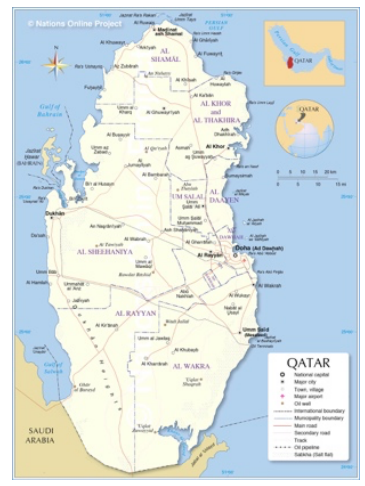

Figure 1. Geographic Map of Qatar

This study engaged the participation of chosen Filipino frontline workers in Qatar. The participants were selected after obtaining approval from them and the relevant authorities in the research locale. The following criteria were used to select participants: (a) a Filipino frontliner who is working in Qatar for at least 2 years and (b) in the age range of 21-60 years old. In doing the research, protocols were followed to get the consent of the participants and authorities involved in order to conduct the research.

\subsection{Data Collection and Ethical Consideration}

It is important to follow ethical guidelines in research as it is a critical component of the study's success. Obtaining the data and information are needed to conduct the study. In the study, an interview was conducted with the Filipino frontliners in Qatar in order to gather data and needed information. The questionnaire was prepared by the researchers to guide them on the questions to be asked to the participants. The questionnaire consists of 28 questions that have answered how Filipino frontliners deal with stress, the most recognized struggles that they experience, and their common adjustments. The research gathered the participants' experiences for the study by asking the consent of the participants to join the interview and permission to record the conducted conversation. All information that the researchers have gathered are taken as highly confidential at all times. The researchers also made sure that all of the researchers kept the identity of the participants' background, personal information, and the data that the participants had shared to be private to protect their confidentiality, making sure that it is well maintained and secured. The importance of making sure to keep the privacy of the participants is needed to ensure the ethical standards of the research.

\subsection{Data Analysis}

After gathering all of the information and data needed, analysis and thematizing were made. Since the interview was done through the use of video conference and a voice recording, the researchers transcribed all 
of the participants' answers systematically. After the emic to etic transcription, the researchers proceeded in doing the cool analysis to modify the responses in a comprehensible way and to summarize all of the participants' answers.

The warm analysis was then formed where the grouping of similar responses and the making of the themes were done. The themes that were formed are (1) Work Constraints, (2) Work Impact, and (3) Coping Strategies. Formulating these themes helped the researchers to form the dendrogram based on the warm analysis.

A thought unit was created that led to the forming of the simulacrum. The simulacrum was formed based on the analysis of the researchers about the lived experiences of the challenges and adjustments of Filipino frontliners in Qatar.

\subsection{Simulacrum}

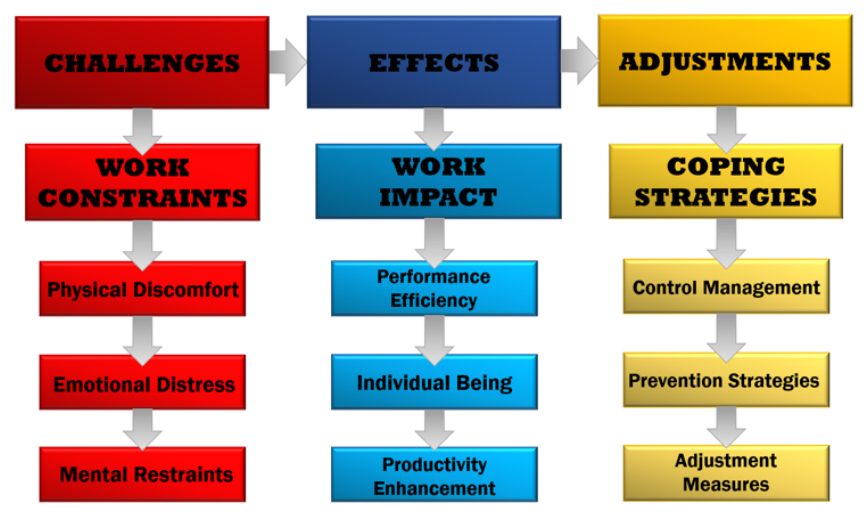

Figure 2. Challenges and Adjustments of the Filipino Front liners

The arrow from left to right signifies the themes arranged in order that show the challenges, effects, adjustments of the frontliners. Arrows that are pointing below the corresponding themes are the sub-themes. The theme 'Work Constraints' is represented by the color red for problems and danger. This focuses on the physical discomfort, emotional distress, and mental restraints of the frontliners. While the color blue was chosen for 'Work Impact' signifying stress and sadness. This section focuses on the consequences of stress on frontline workers' performance efficiency, individual being, and productivity enhancement. Yellow, the hue of optimism and hope, is used to signify coping strategies. The emphasis is on how the frontliner copes with and adjusts to various situations which focuses on control management, prevention strategies, and adjustment measures of the frontliners.

\section{Results}

The study describes the lived experiences of Filipino frontliners in the State of Qatar. The world's current situation, Covid pandemic, emphasized the hard work that the frontliners do. The line of work of the frontliners contain challenges, which come along with adaptations in their current situation. Work Constraints, 
Work Impact, and Coping Strategies were the three key themes that emerged from the responses of the participants.

\section{Work Constraints}

In the workplace, constraints are conditions or features that prevent employees from achieving their full potential by preventing them from using their full abilities or effort. Frontline personnel are confronted with several limitations especially due to a variety of factors. This demonstrates that scheduling, change of duties or assignments, language barriers, mood swings, and sickness are all sources of stress for frontline workers. Work constraints focused on the factors that limit frontline workers' ability to perform to their full potential and capacities. These limitations, such as frantic scheduling, have an impact on the personal life of frontline workers.

\section{Physical Discomfort}

The physical condition of front liners during work was affected by a number of factors. Long hours of working, having too many patients, a hard workload, and being unwell have all resulted in poor energy, headaches, and muscular discomfort for frontline workers. Schedule, change of duties or assignments, mood fluctuations, and sickness have all caused stress on the frontliners, affecting their productivity. The following participants expressed:

"During long hour shifts such as twelve-hour shift headaches and muscle pain in the likes are very common." (P1)

This assertion was backed up by two more comments that illustrate how a variety of circumstances impact frontline workers' physical condition during their shift.

"I experienced headaches when there were too many patients, also when we have to extend our working hours." (P2)

This statement described that stressed individuals' pressure is the surface of their frustrations, and when it is aroused, it leads them to be unproductive.

"One of the stress factors is a stressful patient, especially during this pandemic. Right now, regardless of healthy patients or irrespective of any condition a person has, we need to wear our proper PPE, that's very uncomfortable and you will really perspire. Also, we are not allowed to go on our vacation to the Philippines." 
Long shifts and congested uniforms drained their physical capacities, leaving them unable to address their grievances effectively, leading them to overlap, resulting in physical weariness.

\section{Emotional Distress}

Frontline workers' emotional conditions were affected by a variety of factors, resulting in job limits. The majority of frontline personnel experienced tension and anxiousness, while some attempted to overcome these unpleasant emotions. Being paranoid, sensitive, and fatigued were all offered as examples of distinct emotional strains. One confirmatory statement stated,

"There are times that when I'm dealing with a patient who has a problem with his/her body, I feel a little pity for them. It affects me personally so I need to show sympathy with the patient." (P5)

Another comment backed up this assumption, demonstrating how a range of events affects frontliners' emotional state during their shift.

"It's very important that you are not moody and that you have really emotional stability. If you will really be stressed, you will not be patient, you'll explode. You will have self-control and patience and presence of mind and also be flexible to understand the situations of every individual." (P6)

The emotional state of frontline workers was affected by their patients' experiences and present situations, leaving them unable to adequately address their concerns, leading to battles and emotional pressures.

\section{Mental Restraints}

One of the limits mentioned is the mental restraints that frontline personnel encounter. Their way of thinking has been influenced by their schedules, demanding supervisors, individuals they meet, and their work. The mental health of frontline workers was influenced by a variety of work-related issues. One participant said,

"Yes, it happens, sometimes in my case when I am stressed, I tend to lose focus at work and worry a lot and overthink." (P1) 
In addition, two more comments illustrated how a variety of circumstances can affect a frontline worker's mental condition during the shift.

"I recognize stress when I can't function well and can't give my 100\% to the outputs anymore. It affects your work, decision-making, and your output." (P2)

This highlighted how weariness has an impact on frontline workers' mental health. Individuals that are under pressure make rash decisions, which has an impact on their outcome.

"When I'm having migraines, I can't really think well and it affects my scanning technique." (P5)

Frontline employees' mental health was impacted by a range of work-related challenges, leaving them unable to appropriately address their concerns, resulting in conflicts and emotional strain.

\section{Work Impact}

In the working environment, work impact is focused on the effects of stress on the frontliners' performance at work, their personal self, and their productiveness. Work Impact further discusses how frontline workers adjust to the working environment with various situations, and difficult scenarios such as power outages and language barriers. To add, the change of schedules, long working hours, and sudden change in routines were a also few of the challenges that the frontliners faced.

\section{Performance Efficiency}

The frontliners' way of performing a task at work during a difficult situation. Frontliners explained that the physical environment has affected their performance with the various personalities of people they encounter and unavoidable situations such as electricity outages. A few have also mentioned that they have encountered errors in their work due to stress.

"One time I worked in the office and then a brownout happened for just a short period of time. It affected me because I can not function and do the work. I only had to sit and wait for it to come back." (P2)

This claim proves that frontliners are unable to work efficiently and effectively when encountered with disruptions in the working environment.

"If you go to work with enough rest, enough food and refresh your mind and of course prayer we will not have errors." (P6)

As the participant explained, with enough energy and proper mindset; frontliners can work more productively and make fewer mistakes. 


\section{Individual Being}

As for individuality, this focuses on the frontliners' reaction and emotional response to difficult scenarios such as the language barrier. Frontliners have mentioned that they become sensitive and temperamental when stressed and pressured. Their emotional response to adjusting to new work schedules often led to overthinking and being sentimental.

"I always felt tired, I am afraid, and I tend to overthink, and it burns me out." (P1)

When frontliners were feeling stressed, their emotions and state of mind were being affected. Thus, when responding to something new or different, frontliners tend to overthink and become sensitive.

“Our job isn't stressful besides having a hard time communicating to our superior because of the language barrier." (P4)

This response proved that the difficult scenarios encountered by frontliners such as language barriers and other minor problems can stress them out.

\section{Productivity Enhancement}

The frontliners productivity during a certain time of the day. Most frontline workers prefer working a day shift since they are more productive in the morning however, others can work fine either in the morning or night shift. It was also mentioned how their emotional stability affected their performance at work. Frontliners mentioned that being happy and in the right condition makes the front liners more productive.

"I prefer day shift rather than night because I can't last long without sleep." (P2)

Each frontliner has its own preference for working either day shift or night shift. However, most frontliners have said that they prefer working in the morning as it makes them more active.

"Of course when you're happy, you're productive and when you are stressed, it affects your work." (P5)

As mentioned by the frontliner, being happy and in the right condition lets them accomplish tasks productively.

\section{Coping Strategies}

Coping strategies distinctly focused on the strands on how the front-line workers manage the changing environment around them every day and was also the mental process of a person in dealing with stressful events. This is a deliberate response to the difficulties a person faces. The coping strategies in their work environment enabled them to control their emotions and also helped them contain their peace of mind. These strategies prevented them from stressing and overthinking during the times where their work environment changes gradually. 


\section{Control Management}

The frontliners ability to sustain their emotions in difficult scenarios. It is their control to not physically act on negative based emotions and also the ability to control themselves mentally during stressful situations. Based on one remark that backs this up, "There are times I am being temperamental, sensitive and irritated by small things, however when I am at work, I am able to manage my work and I am decent with my workmates and my boss."(P1)

This statement is supported by two further responses that explain the situation on how frontliners are able to control themselves.

"I remained calm, smiled at all times, and when it's out of my control I immediately call my supervisor for help. "(P4)

The response of the participant proves that frontliners have the capability to remain in an orderly manner when facing stressful situations. Hence, one participant indicates one of their coping strategies when it comes to having control.

"Sometimes I just walk and rest for a few minutes, inhale and exhale, making sure that I have time to relax and then after fifteen minutes I go back to working again. "(P2)

\section{Prevention Strategies}

Prevention is the way on how frontliners cope by preventing events that could affect them in the long run. Frontliners prepared for potential long-term occurrences by amassing resources that could help prevent a severe impact on them. Here follows a confirmatory statement stating, "I just prevent this situation of stress by putting and by giving all my trust on our creator I give it all to God."(P6)

The statement was supported by two more answers that indicate how frontliners prevent hard situations that might affect them in the long run.

"You need to be able to manage your schedule properly. You need to have proper day-offs and take a rest or spend some time with your family. "(P2)

Furthermore, this implied that being focused on the mental state of oneself helped the frontliners

take a break and stop future stressful situations. As said by a participant, being able to reconnect with loved ones helped in preventing stressful situations.

“Going out to dine-in restaurants, café's and conversation with my husband helps me a lot."(P1)

Thus recognizing the responses, having the right coping mechanism like turning to God for help, being organized, and spending time with family helps the frontliners in preventing future stressful scenarios. 


\section{Adjustment Measures}

As to adjustments of the frontliners, they adjusted by hoping that everything will be okay in the end. Staying optimistic helped the frontliners a lot especially during difficult times. Others adjusted by discussing with their boss about their work schedules so that it would help them ease into it naturally or at least prepare themselves for it.

According to one confirmatory statement, "Adjusting is difficult but I adjusted by being optimistic, positive and saying to myself that everything will be alright and it will end soon."(P3)

This assertion was backed up by two further answers that clarify how their coping strategies are with adjustments.

"For me, I was able to adjust naturally. I kept praying and thinking that everything will be okay."(P1) The answer shows that being optimistic and praying to God helps the frontliners in adjustments in their work environment. When adjustments arise some were able to talk about it with someone and this helped them adjust properly as said by a participant:

"I talk to my supervisor or the person in charge with the schedule to put me in the morning so I can adjust to it." (P5)

Considering the participants' statements, each has their different ways of coping with the adjustments that occur in their workspace.

\section{Discussion}

Frontliners in Qatar encountered different challenges in their workplace that affected their lives which they made a full effort to adjust. The main objective of this study was to determine the different challenges that the frontliners have encountered and their adjustments to those situations. The frontliners have stated their experiences in the challenges and adjustments in their workplace and personal lives.

\section{Work Constraints}

Work Constraints mean that the realization of full performance was prevented due to the situations of the work environment. Frontliners described the stress factors that affect them in the Physical, Emotional, and Mental side of their work. The researchers included the terms Physical Discomfort, Emotional Distress, and Mental Restraints as the sub-themes for Work Constraints, as the data fits the responses of the frontliners.

The sub-theme Physical Discomfort showed many different factors that affected the physical condition of the frontliners that lead to full body fatigues. In the Emotional Distress sub-theme, the results showed that the frontliners recognized stress and discomfort feelings. Fear, self-isolation, and lack of access to proper equipment contributed to the emotional stress of the frontliners (Bennett, 2021). Lastly, the sub-theme Mental 
Restraints showed the results that the frontliners' mental health is influenced by a variety of work-related issues namely, excess demands and fear of the crowd. Frontliners received anxiety, depression, post-traumatic stress disorder, and increased substance abuse, as they worked through the COVID-19 pandemic that could lead to a rise in compensable mental health claims down the road (Childers, 2021).

\section{Work Impact}

The effects of stress on frontliners' work performance, personal well-being, and productivity were the focus of Work Impact. It discusses how they deal with different situations in the working environment. The sub-themes included in Work Impact are Performance Efficiency, Individual Being, and Productivity Enhancement.

The sub-theme Performance Efficiency discussed the performance of frontliners in their work. Frontliners who took good care of themselves did not cause an error while working caused by stress. For its workforce to efficiently manage COVID-19 cases, front-line workers need to be physically and mentally healthy to face any challenge (Sim, 2020). Frontliners who are productive and efficient in their performance at work; focused on their health. In addition, the physical environment has an impact on their work performance as well. Front liners are unable to work efficiently when encountered with disruptions in the working environment such as electricity outages.

The Individual Being sub-theme focused on their personal lives. Frontliners became sensitive and temperamental when stressed and pressured. Burnout, overthinking, and becoming sentimental were among the negative effects on frontline workers' emotional and mental well-being.

Lastly, the Productivity Enhancement sub-theme showed the motivations of frontliners in different circumstances. It was discovered that most front liners prefer working with a day shift as they are more productive in the morning. Furthermore, their emotional stability affects their performance at work as being in the right condition makes them more productive. People who are creating positive feelings are more likely to devote more time to more creative work, which may lead to increased productivity (Isen, 2006).

\section{Coping Strategies}

Coping Strategies talks about how the front-line workers managed their mental, emotional, and physical health. It defines how they deal with the situation to overcome a certain problem or difficulty in their life. The sub-themes emerged in the Coping Strategies were Control Management, Prevention Strategies, and Adjustment Measures.

The sub-theme Control Management dealt with how the frontliners take everything under control that involves their physical, mental, and emotional health. With the different situations that the frontliners have to face through, they mentioned that they have to have self-control in body and mind. As mentioned by Søvold et al. (2021) Practicing self-care can also help healthcare workers create some structure and predictability amidst chaos and uncertainty and make them able to manage high levels of stress in more constructive ways. Even with the small things dealing with their job like time management, the language they are speaking, frontliners controlled everything to make their job productive and to cope with stress and difficulties. 
The sub-theme Prevention Strategies discusses the frontliners' strategy to avoid any negative behavior that will affect their work. Smida et al. (2021) specified that gaining the skill of optimism can assist in confronting stress or setback, can help to overcome failure in particular events, and strengthens self-efficacy and resilience. The participants revealed that they had to deal with the unruly behavior and compulsive attitude of the patients in their workplace, but frontliners prevented this by showing more patience and by just taking time to rest. Lastly, the sub-theme Adjustment Measures shows how the frontliners adjust in every situation in their workplace. The researchers discussed how the frontliners adjust based on their time, people that they interact with, physical energy, and certain situations that they should be aware of.

\section{Conclusion}

Frontliners continue to work productively while they experience difficult situations. Based on the results and discussion of the research, frontliners encounter stressful events such as physical discomfort, emotional distress, and mental restraints while experiencing emotional conditions. However, these situations allow them to create new management strategies and adjust to different scenarios. Frontliners are able to develop new coping strategies that help improve their mental, emotional, and physical health as well.

Going to work has exposed frontline workers to unprecedented levels of stress and pressure, jeopardizing their mental, physical, emotional, and even social well-being. While organizations and health administrators must make many of the initiatives to minimize stress and care for frontline workers, frontline workers can also take measures to cope with stress. Frontline workers may feel annoyed, helpless, exhausted, fatigued, or out of focus in some situations, but it is fair to take a break for them to acknowledge their emotions. Communication with the frontliners' loved ones or colleagues, where they may openly discuss their work-related stress, can help reduce the feeling of being worn out. It is also important for them to recognize that frontliners are playing a critical part in a heavy and risky job, and that they are doing the greatest they can with the capabilities they have. On the other hand, there are situations front-line workers do not have control of in which they need to accept. Being a frontliner is a difficult yet courageous job, thus it is essential to have a healthy body by keeping a daily routine. To begin, eat healthy meals, drink vitamins, schedule time to maintain an exercise routine, make time for a hobby, rest when there is an opportunity, and last, take some deep breaths.

If frontliners need a shoulder to cry on or a quick escape, reach out to let them know that someone is available to listen and communicate with them. Frontline workers will receive the support and comfort they need from their loved ones as a result of doing thoughtful actions. All of the efforts of the frontliners must be appreciated during difficult times like a pandemic, as it has a tremendous influence on them. Staff members will be more productive if their responsibilities are distributed equitably, work hours are adhered to, and they are given ample relaxation. In light of these factors, the frontliners will be able to perform at their best. It is also important for organizations to provide workers with the healthcare they deserve and to ensure their safety while in the workplace. Frontline workers will feel validated and confident in their abilities if their voices and dedication are valued in the workplace and community.

Future studies will gain from this research as it will provide them with new information and ideas. Future researchers can use the results of this study to extend the idea of how to help frontline employees who are facing challenges, whether or not a pandemic is present. Recommendation would be a further 
interview with front liners without a pandemic. Moreover, this study outlined the common issues that frontline employees encountered, which future researchers may use to compare and contrast the challenges that frontline workers faced then versus in the future. In order to increase the knowledge of this research, future researchers are recommended to choose more participants from outside the medical industry. The findings of this study can be used by researchers in the future to acquire a better understanding of frontline employees.

Despite the fact that enough data was collected to address the research study's question, there were also limitations encountered. The researchers had a difficult time choosing volunteers because the majority of the participants work in the medical field, despite the researchers' desire to cover all occupations affected by the epidemic. Due to the hectic schedules of frontline employees, it was challenging for the researchers to find participants who could make time to engage in an interview for this research. Since the study only focused on Filipino front-line workers in Qatar, future researchers may want to consider expanding the findings of this study to include Filipino front-line workers globally. 


\section{References}

Bennett,Hannah.,(2021). Psychological Effects of COVID-19 on Frontline Workers. https://www.domesticpreparedness.com/healthcare/psychological-effects-of-covid-19-on-frontlineworkers/

Bloor, M., \& Wood, F., (2006). Keywords in Qualitative Methods. Sage https://orca.cardiff.ac.uk/93247/

Buhat, C. A. H., Torres, M. C., Olave, Y. H., Gavina, M. K. A., Felix, E. F. O., Gamilla, G. B., Verano, K. V. B., Babierra, A. L., \& Rabajante, J. F. (2020). A mathematical model of COVID-19 transmission between frontliners and the general public. https://www.medrxiv.org/content/10.1101/2020.03.27.20045195v1. full-text

Childers,A.,(2021). Fatigue, stress take toll on frontline workers. https://www.businessinsurance.com/article/20210304/NEWS08/912340080/Fatigue,-stress-take-tollon-frontline-workers-COVID-19-coronavirus-pandemic-

Crossman, A, (2020). An Overview of Qualitative Research Methods. ThoughtCo.https://www.thoughtc o.com/qualitative-research- methods-3026555

Gamio, L. (2020). The Workers Who Face the Greatest Coronavirus Risk. The New York Times. https://www.nytimes.com/interactive/2020/03/15/business/economy/coronavirus-worker-risk.html

Isen, A.M., Reeve, J. (2006) The Influence of Positive Affect on Intrinsic and Extrinsic Motivation: Facilitating Enjoyment of Play, Responsible Work Behavior, and Self-Control. Springer Link. Motiv Emot 29, 295-323 (2005). https://doi.org/10.1007/s11031-006-9019-8

Neubauer, B.E., Witkop, C.T. \& Varpio, L. (2019). How phenomenology can help us learn from the experiences of others. Perspect Med Educ. https://doi.org/10.1007/s40037- 019-0509-2

Sim, M. (2020). The COVID-19 pandemic: major risks to healthcare and other workers on the front line. Occup Environ Med. doi:10.1136/ oemed-2020-106567

Smida, M., Khoodoruth, M. A. S., Al-Nuaimi, S. K., Al-Salihy, Z., Ghaffar, A., Khoodoruth, W. N. C., Mohammed, M. F. H., \& Ouanes, S. (2021). Coping strategies, optimism, and resilience factors associated with mental health outcomes among medical residents exposed to coronavirus disease 2019 in Qatar. Brain and Behavior, 11(8). https://doi.org/10.1002/brb3.2320

Søvold, L. E., Naslund, J. A., Kousoulis, A. A., Saxena, S., Qoronfleh, M. W., Grobler, C., \& Münter, L. (2021). Prioritizing the Mental Health and Well-Being of Healthcare Workers: An Urgent Global Public Health Priority. Frontiers in Public Health, 9. https://doi.org/10.3389/fpubh.2021.679397

Sritharan, J., Jegathesan, T., Vimaleswaran, D., \& Sritharan, A. (2020). Mental Health Concerns of Frontline Workers During the COVID-19 Pandemic: A Scoping Review. Global Journal of Health Science, 12(11), 89. https://doi.org/10.5539/gjhs.v12 n11p89 